Relations industrielles

Industrial Relations

\title{
Written Communications for Business Administrators, Robert D. Hay, Holt, Rinehart and Winston Inc., Toronto, 1965, 487 pages
}

\section{Ronald Pleau}

Volume 20, numéro 3, 1965

URI : https://id.erudit.org/iderudit/027600ar

DOI : https://doi.org/10.7202/027600ar

Aller au sommaire du numéro

Éditeur(s)

Département des relations industrielles de l'Université Laval

ISSN

0034-379X (imprimé)

1703-8138 (numérique)

Découvrir la revue

Citer ce compte rendu

Pleau, R. (1965). Compte rendu de [Written Communications for Business Administrators, Robert D. Hay, Holt, Rinehart and Winston Inc., Toronto, 1965, 487 pages]. Relations industrielles / Industrial Relations, 20(3), 578-579. https://doi.org/10.7202/027600ar

Tous droits réservés (C Département des relations industrielles de l'Université Laval, 1965
Ce document est protégé par la loi sur le droit d'auteur. L’utilisation des services d’Érudit (y compris la reproduction) est assujettie à sa politique d'utilisation que vous pouvez consulter en ligne.

https://apropos.erudit.org/fr/usagers/politique-dutilisation/ 
Bref tous ces exercices peuvent contribuer ò rendre plus intéressante et profitable l'étude de l'économique ce qui peut avoir pour résultat de rendre les étudiants mieux informés et plus responsables selon les outeurs.

\section{Ronald Pleau}

\section{Managerial Behovior: Administration in Com-} plex Organizations, par Leonard R. Sayles, McGraw-Hill Book Co., Toronto, 1964, 269 pages.

Le professeur Sayles est déjà connu par ses trovaux sur le leodership syndical, le compartement des groupes de travoil, sur l'incompatibilité qui peut exister entre le développement de l'individu et la bureaucratie maderne. Ce dernier volume est plutôt une tentative visant à décrire en des termes opératoires ce que font exactement les dirigeants de la grande entreprise. Cet essai s'éloigne passablement de la théorie classique qui s'ocharne à décrire le rôle de l'odministrateur vio les grandes fonctions administrotives. II s'éloigne oussi de l'école des * relotions humaines en déloissant l'étude des petits groupes qui oeuvrent au bas de la pyramide sociale pour pénétrer dons les arcanes de la houte direction.

Cette pénétration s'est faite par l'étude des activités commandées et intégrées par la technologie particulière dे chaque entreprise, par l'étude du contenu, de la fréquence des échonges qui se font entre les dirigeants ou sein de l'organisation et d l'extérieur. L'administrateur oeuvre au centre de ces contocts journaliers. Son rôle consiste à gagner l'assentiment ou à rechercher les conseils précieux de ces confrères situés ou même palier que lui ou encore à des niveaux différents. Dans ce contexte, les contacts latéraux occoparent plus de $80 \%$ du temps des dirigeants. Cette conclusion invite à repenser la théorie traditionnelle qui se centre sur la nature et la préservation de l'autorité, sur l'importance des relations entre supérieurs et subordonnés.

L'auteur établit une typologie de ces relotions latérales où le rôle de l'administrateur consiste ò composer ovec les individus en vue de la réalisation d'objectifs communs.

Cette perspective laisse volontairement dans l'ombre d'autres aspects du rôle des dirigeants. En plus de marchonder, coordonner et intégrer, les administrateurs doivent discuter en termes techniques, financiers, prendre des risques, élaborer des politiques, etc.

Cependant, ce livre demeure une contri- bution outhentique à cette discipline encore jeune qu'est l'administration des entreprises.

Laurent Bélonger

Written Communications for Business Administrators, Robert D. Hoy, Holt, Rinehort and Winston Inc., Toronto, 1965, 487 poges.

En partant du principe sur lequel est basé son volume à sovoir que plus une personne est hobile do communiquer plus son odministration est effective, l'auteur divise son exposé en trois parties principoles dons lesquelles il s'en tient oux formes de communications écrites dont se servent le plus souvent les hommes d'offoires.

Lo première est consacrée à la correspondance d'affaires dont se servent les administroteurs pour atteindre leurs objectifs. Ainsi une fois que ces derniers connoissent bien la procédure de bose des communicotions, le concept de la correspondance d'offoires de même que le pourquoi, les ovantages et désavantages de ces lettres, ils doivent passer par trois étopes importontes pour devenir des gens compétents en cette motière.

L'une consiste d̀ reconnoitre que certains standards psychologiques portent directement sur la composition des lettres d'affaires lesquels peuvent se diviser en deux groupes principoux, ceux en ropport avec une pensée claire et ceux correspondant à l'attitude du compositeur. On appuie surtout sur la considération du lecteur, laquelle est l'aspect humain des communications et la clé du succès dans la transmission d'un message, sur l'adoption d'un ton positif et de l'emploi de la courtoisie dons la composition de ces lettres, sur la foçon d'arriver d̀ convoincre par ce medium et à créer de l'intérêt chez le lecteur. La concision, la précision et l'usage adéquat de la ponctuation sont des qualités également requises pour une borne composition.

Lo seconde étope concerne le plan de lo lettre, Comme une lettre est composée dans un but précis, il importe de considérer tous les focteurs importants de développer pour arriver à bôtir un plan effectif. L'outeur nous fournit à ce sujet des exemples de plans concernant cinq situations types de camposition de lettres d'offaires.

Enfin, dans une troisième étape il est essentiel pour qui compose ses lettres de bien comprendre et apprécier leur apparance 
physique cor l'impoct que celle-ci crée sur l'impression première du lecteur peut faire la différence entre l'acceptation ou le rejet du messoge par ce dernier.

La seconde partie du volume porte sur lo méthode scientifique d'écrire des rapports. Robert D. Hay nous présente dans quelques chapitres une procédure d'analyse d'un problème consocrant un chapitre à chacun des thèmes suivants: collection des données ou de l'information, organisation et interprétation de celle-ci pour arriver ensuite da la rédaction du rapport lui-même.

Les communicotions graphiques font ensuite l'objet d'une étude dans un chapitre qui traite des standards de présentations des données quolitatives et quantitatives.

Enfin, même si le volume porte sur les communications écrites, cette seconde partie se termine par l'étude des méthodes orales de présentation des informations dे un petit groupe de gens.

Ce n'est que dons une troisième partie beouccup élaborée que sont décrits et étudiés les différents problèmes auxquels fait foce un administrateur qui emploie les conoux internes de communications de son orgonisation, por la présentation des principes de communication entre employeur et employés et l'étoblissement des politiques et règlements écrits qui focilitent lo coordina. tion ò l'intérieur de l'organisation.

Ce volume, donc, paroit de lecture intéressante et utile tant à couse de la variété des thèmes qui y sont traités que par la formule odoptée. En effet, chocun des chapitres est accompagné de quelques problèmes pratiques por lesquels l'outeur fait participer le lecteur et met à l'épreuve les connaissonces qu'il vient d'acquérir par la lecture des pages qui précèdent.

\section{Ronald Pleau}

The Economics of Health and Medical Care, Proceeding of the Conference on the Economics of Health and Medical Core, The University of Michigan, Ann Arbor, Michigon, 1964, 321 poges.

De plus en plus les économistes manifestent un certain intérêt pour la recherche dans le domaine de la sonté et des soins médicoux apportant ainsi une contribution des sciences sociales ò ce genre de problème. Ceci est dû à l'émergence des questions de politique sociale concernant la relation entre la croissance économique et la santé dans les poys en voie de développement et oux conséquences des récentes innovations dans l'organisation et le financement des soins médicaux.

C'est ce qui constitue en quelque sorte le sujet d'étude du rapport d'un congrès tenu à l'Université du Michigan et groupant des économistes intéressés à travailler à lo dénition du rôle de l'économiste dans la recherche sur les problèmes de la sonté et des soins médicoux.

A la suite d'une introduction nous expliquant la raison d'être d'une discipline de l'économique de la santé, résultat des efforts de recherche sur les sources de la croissance économique, et nous donnant une énumération des problèmes que pose l'économique médicale, le développement de cet ouvrage nous présente trois groupes principaux d'exposés élaborés plus spécifiquement par les panélistes.

Les premiers concernent les méconismes par lesquels les services santé et leurs utilités constituantes sont coordonnés et agencés ou moment et à l'endroit précis pour ré. pondre oux exigences des consommateurs. Ils mentionnent où est rendu une analyse économique détaillée de ces questions oux Etats-Unis au moment du congrès. Partant de l'impact sur les coûts et les services de prévention des tests actuels d'exigibilité pour. le soin médical public dans les villes, ils vont jusqu'd analyser les soins de lo sonté en tant que variable de la croissance économique. Suivant son schème d'analyse habituel, l'économiste tourne surtout son ottention vers les questions d'efficacité de l'emploi des ressources. Ainsi on fait des comparaisons détaillées sur les ressources par unité de service.

Un outre groupe d'articles traite des questions de mesure des facteurs qui déterminent la demande, de mesure des prix et des changements de coût et leurs implicaticns sur les primes d'assurances.

Enfin, un troisième secteur est examiné, soit celui des différentes facettes des programmes de santé et du développement économique. Les auteurs passent d'un essai de mesure de l'investissement dans les fonds de santé de la force de travail à la planification familiale en tant que composante des programmes de santé dons les pays sous-développés et sur-peuplés.

A portir enfin des idées exprimées et des conclusions atteintes por les groupes de discussion de ce congrès, Jerome Rothemberg bôtit, en guise de résumé, un agenda de la recherche pouvant possiblement être entre- 